\title{
Stealth Adapted Viruses: A Bridge between Molecular Virology and Clinical Psychiatry
}

\author{
W. John Martin \\ Institute of Progressive Medicine, South Pasadena, CA, USA \\ Email: wjohnmartin@ccid.org
}

Received 12 August 2015; accepted 26 September 2015; published 29 September 2015

Copyright (C) 2015 by author and Scientific Research Publishing Inc.

This work is licensed under the Creative Commons Attribution International License (CC BY). http://creativecommons.org/licenses/by/4.0/

(c) (i) Open Access

\begin{abstract}
Cytopathic "stealth-adapted" viruses bypass the cellular immune defense mechanisms because of molecular deletion or mutation of critical antigen coding genes. They, therefore, do not provoke the inflammatory reaction typical of infections with the conventional viruses from which stealth adapted viruses are derived. Stealth adapted viruses establish persistent, systemic virus infections, which commonly involve the brain. The brain damage can cause major mood and cognitive disorders, fatigue, seizures and various manifestations of an impaired autonomic nervous system. Symptoms can also result from: 1) induced autoimmunity, 2) antibody formation against virus antigens, 3) virus-induced cellular damage to non-brain tissues and 4) induced heightened overall immune reactivity, such that normally unrecognized components of the virus begin to become targeted by the cellular immune system. This last mechanism is relevant to the reported neurological and psychiatric adverse effects of vaccination in certain individuals. It is also appropriate to consider the infectious component of stealth adapted virus infections since family members and others may be at risk for becoming infected.
\end{abstract}

\section{Keywords}

Stealth Adapted Viruses, Cytomegalovirus, African Green Monkey Simian Cytomegalovirus, SCMV, Encephalopathy, Polio Vaccine, Chronic Fatigue Syndrome, Autism, Schizophrenia, Alzheimer's Disease, Delusion, Schizovirus

\section{Overview of Traditional Neurology and Psychiatry}

Dysfunctional brain syndromes are traditionally viewed as comprising two distinct groupings of illnesses; neurological and psychiatric. Neurologists mainly address diseases that can be attributed to discrete anatomic lesions, with readily elicited physical signs pertaining to the affected region of the brain. Although the therapeutic 
options are usually limited, the causes of these illnesses have a rational basis in terms of defined neuro-anatomical lesions. As opposed to neurologists, psychiatrists and other mental health personnel, mostly address diseases lacking precise anatomic localization or biologic explanation. These diseases are expressed in terms of varying degrees of altered emotions, behaviors and cognitive processes; functions that are viewed as expressions of the "mind" rather than of the "organic brain". The availability of mind-altering drugs has helped shift the therapeutic emphasis for these diseases from simply trying to coerce the patient to change his or her ways (psychotherapy) to the somewhat more successful (if still empirical) psychopharmacological approach. The use of therapeutic drugs in psychiatry is predicated on the assumption that patients have an underlying disturbance in neural metabolism that can be at least partially restored pharmacologically. The etiology of the presumed "chemical imbalance” is rarely addressed and often assumed to be a result of an inappropriate behavioral adaptation to life stressors. This paper provides an alternative explanation for many psychiatric illnesses; one that is based on altered brain function resulting from infection with stealth adapted viruses.

\section{Spatial Distribution of Normal Brain Function}

The brain is unique among the body's organs in the spatial distribution of its many functions. Unlike other organs, damage to one area of the brain cannot be readily compensated for by heightened activities in other brain areas. Moreover, individual components of the brain participate in complex neural networks, which can subserve a variety of integrated functions. Even minimal damage to neurological tissue has the potential for profound symptomatic effects; compared to the effects of limited cellular damage occurring in extra-neural tissues. Not only is the brain tissue spatially complex, it is hampered by the inability of mature neuronal cells to replicate and to replace neurons damaged by either illness or normal senescence.

\section{Assessment of Brain Function}

The brain is responsible for motor, sensory, autonomic and cognitive functions. It also determines personality, mood, self-perception and social interactions. Assessment of gross deficits of sensory and motor functions is readily achieved in routine neurological examinations. Psychiatrists rarely, if ever, employ testing for more subtle sensory and motor changes, and for possible derangements of the autonomic nervous system. Many neurologists have also disregarded such tests as only providing inconsequential “soft signs”. Complex assays, such as tilt-table testing for orthostatic hypotension can provide a quantitative measure of a specific autonomic function, but are unsuitable for everyday clinical practice. Neuroimaging techniques, such as computerized EEG, PET scans and functional MRI can also provide measures of brain activity but are also unsuitable for routine psychiatric practice. Furthermore, the etiological foundations for the minor imaging changes that have been seen in psychiatric patients are not yet established. Neuropsychiatric testing for minor personality disorders and for mild cognitive impairments requires an in-depth knowledge of the individual's pre-illness performance; information which is not generally available. One-time testing will, therefore, usually not reveal the early changes in personality or cognitive abilities that patients themselves or their friends may perceive.

\section{Diagnostic Labeling of Psychiatric Illnesses}

In spite of the shortcomings in assessments of many brain functions, psychiatrists have managed to categorize psychiatric illnesses into distinct clinical entities by grouping symptoms into a variety of syndromes [1]. These groupings obscure the fact that many symptoms are common to various disease categories. Moreover, the naming of an illness tends to overlook the considerable variability in symptoms, and especially in their relative severity between patients and even in a single patient over time. The lack of true diagnostic precision in reflected in such terms as "co-morbidity" and "borderline condition.” The assumption that different syndromes have different underlying etiologies has also hampered efforts to find common causes of mental illnesses.

\section{Etiology of Psychiatric Illnesses}

In a similar way that diagnostic labels have tended to artificially sub-divide a spectrum of neuropsychiatric illnesses, the proponents of various etiologic theories have also tended to be exclusive rather than inclusive. The notion that organic brain illness is genetic, infectious, autoimmune or toxic, precludes the known interactions between all of these components. The aging process itself can slowly erode the limited functional reserves that 
may have survived an earlier insult. This can lead to a delay in the clinical expression of an illness until years after the initiating event has occurred. Of the four etiologic categories listed above, an infectious cause has the promise of being the most readily targeted for therapy, as well as having the added concern of being potentially transmissible between individuals. Chronic viral infection of the brain can present in many different ways depending simply on its localization to different regions of the brain and on the preexisting functional capacity levels prior to infection. Chronic infections can also render individuals susceptible to normally tolerated environmental factors and to other stressors of brain function [2].

\section{Viruses and Psychiatric Illnesses}

The digression of psychiatry from basic molecular biology is seen in the minimal attention currently given to the potential role of viral infections in psychiatric illnesses. Historically, such conditions as encephalitis lethargica, subacute sclerosing panencephalitis, multifocal leukoencephalopathy and general paresis of the insane were belatedly accepted as infectious [3]. The reality of AIDS dementia is also now unquestioned [4]. On the other hand, early attempts to detect viruses in patients with schizophrenia (schizoviruses) and other major psychiatric illnesses, failed to provide convincing and readily reproducible findings [5]. In spite of the availability of more sensitive technologies, such as the polymerase chain reaction (PCR), few psychiatrists are intellectually poised to consider viral infections as a likely cause of their patients' illnesses.

The prevailing model of a viral brain infection is that of herpes simplex virus (HSV) encephalitis. Typically, the patient will present with an acute onset ( $<2$ weeks from the initial symptoms to severe illness); have progressively diminishing level of consciousness; show localizing signs, often to the temporal lobes, on clinical, radiologic and EEG examinations; and have numerous lymphoid cells in the cerebrospinal fluid (CSF) with increased protein levels [6]. Relatively mild meningitis/encephalitis-like illnesses are also commonly encountered in General Practice. If pursued vigorously, serological assays, changes in CSF, and virus cultures of feces will occasionally indicate an enteroviral infection [7]. The illnesses are considered to be short lasting without sequela. The notion of a persisting, sub-acute, non-inflammatory viral encephalopathy is rarely considered clinically or tested for using either viral cultures or molecular probe based assays.

\section{Viral Pathogenesis}

All viruses have the potential to mediate cellular changes by altering the normal metabolic balance within the cell through over utilization of the cell's energy resources [8] [9]. While this can eventually lead to cell death, an earlier cost can be the failure of the cell to perform all of its specialized functions [10]. Continued metabolic drain on the cell can lead to a loss of essential components such as adenosine triphosphate (ATP). A tipping point is reached when there is insufficient ATP to import magnesium into the cell; a required co-factor for ATP activity. Energy starved cells can show foamy vacuolization, swelling and intercellular fusion. Some viruses trigger a more active form of cellular death, called apoptosis, characterized by shrinkage and condensation of cellular components. Herpesviruses, especially HSV and human cytomegalovirus (hCMV), are especially cytotoxic when cultured with normal cells. So too are adenoviruses, influenza, polio and many enteroviruses. Certain human viruses, however, are essentially incapable of inducing a readily detectable cytopathic effect (CPE) in viral cultures on human cells. For example, rubella; hepatitis A, B, C and D; HTLV; Borna and Hantaan viruses are non-cytopathic on cultured cells. Moreover, primary clinical isolates of German measles and mumps viruses induce rather minimal CPE when directly cultured on human cells. For many of these non- or minimally-cytopathic viruses, the major in vivo tissue damage is a consequence of immune activation and lymphocyte killing of the infected cells [11].

\section{Viral Immunity}

The immune system can both reduce and enhance the extent of viral damage. Antibodies can provide an effective blockade preventing viruses from gaining access to normally permissive cells. In particular, antiviral antibodies can help prevent viruses passing through the blood to the brain. Cellular immunity can reduce viral load by destroying infected cells prior to the release of infectious viral particles. On the other hand, cellular immunity against viral antigens or against modified or inappropriately expressed cellular antigens can lead to immune damage of cells beyond that achieved by the virus itself. 
Viruses have evolved various mechanisms to help evade the immune system. One such mechanism is the deletion of the genes coding for the major antigens recognized by the cellular immune system [12] [13]. This mechanism of bypassing the cellular immune defenses has been referred to as a "stealth adaptation.”

\section{Stealth Adapted Viruses}

A corollary of the Clonal Selection Theory of Immunology [14] is that to be effectively recognized, a viral infected cell must present multiple copies of the antigen that is targeted by the responding antigen specific lymphocyte. This requirement restricts the number of different viral antigens, which can be presented to the cellular immune system. Even with large complex viruses, relatively few viral components are targeted for cellular immune defenses [15]. For certain viruses, e.g. hCMV, experimental studies suggested that the complete deletion of the three genes coding for the major viral components recognized by the cellular immune system, would likely yield defective, non-replicating, non-cytopathic viral sequences. The remaining sequences could, however, provide potential building blocks towards the evolution of a cytopathic, non-immunogenic "stealth adapted virus." Potentially, the downsized gene-depleted virus could, for example, form a synergy with a replicating non-cytopathic virus and/or incorporate certain cellular genes by recombination, to yield an atypically structured cytopathic virus. These concepts are embodied in the following definition of stealth adapted viruses:

"Molecularly heterogeneous grouping of atypically structured, cytopathic viruses that cause persistent systemic infection, often with neuropsychiatric manifestations, in the absence of significant anti-viral inflammation. Stealth adaptation is a generic, derivative process in which conventional viruses have lost or mutated the relatively few genes encoding the major antigens normally targeted by the cellular immune system. Stealth adapted viruses typically induce a vacuolating cytopathic effect (CPE) in a range of human and animal cells. The formation, progression, and/or host range of the CPE distinguish stealth adapted viruses from the CPE caused by conventional human cytopathic viruses, including herpesviruses, enteroviruses, and adenoviruses. Additional distinctions can be made on the basis of electron microscopy, serology, and molecular-based studies.”

\section{Origins and Replication of Stealth Adapted Viruses}

Certain stealth viruses contain genetic sequences that are nearly identical to sequences found in African green monkey simian cytomegalovirus (SCMV) [13]. Other regions of these viruses are clearly different from SCMV. Other stealth adapted viruses contain genetic sequences or at least express serological markers related to common human herpesviruses, adenoviruses and enteroviruses. Similar to stealth adapted SCMV, evidence for various cellular genes, have been detected in the DNA and/or RNA fractions of several stealth adapted virus infected cultures. Electron microscopy has also revealed differences between stealth viral cultures in terms of the types and relative abundance of distinctive accumulations of variously structured, complex materials within cells showing the characteristic vacuolated CPE and in the tissue culture medium (unpublished data). An interesting observation is the apparent genetic instability and fragmentation of a stealth viral DNA genome [16]. A potential mechanism of stealth viral DNA replication is through the bridging of viral fragments with long RNA molecules. If so, this scaffolding effect could potentially be inhibited in the presence of short RNA molecules competing with the longer RNA molecule for binding to one of the fragments.

\section{Relevance of the SCMV Origin of Certain Stealth Adapted Viruses}

While stealth adapted viruses have presumably existed for eons, the increasing incidence of many current disease entities is consistent with the introduction of additional stealth adapted viruses through vaccines. Public health authorities have largely ignored the issue of SCMV as a possible contaminant of polio vaccines. This disregard occurred even though in 1972 a joint study by FDA and the vaccine manufacturer showed that all 11 monkey kidney cultures tested using sensitive indicator cell lines showed the presence of SCMV. Only 4 of these isolates would have been detected using the standard detection procedures, which remained in place despite of the above finding (correspondence of polio vaccine manufacturer).

A diverse array of animal cell lines has been used for the many human and animal vaccines that have been developed. It is not unreasonable to suggest that some of these vaccines may have generated stealth adapted viruses. It is also conceivable that vaccine viruses can contribute genetic elements to contaminating herpes and other viruses and that this could facilitate the emergence of replicating, non-immunogenic (stealth-adapted) vi- 
ruses. Once within the human population, stealth adapted viruses can be passed via direct human-to-human contact as well as potentially by interspecies transmission.

\section{Detection of Stealth Adapted Viruses}

The most reliable method for detecting the diversity of stealth viruses is to co-culture the patient's blood with a variety of indicator cell types and observe the cultures for the induction of a transmissible CPE [17]. Typically, rhesus monkey kidney cells and a human fibroblast cell line such as MRC-5 cells are inoculated with the patient's frozen-thawed mononuclear cells and observed for 2 - 4 weeks. Frequent re-feeding of the cultures can help promote the development of the CPE. It is quite unusual $(<10 \%)$ to observe a rapidly developing CPE in blood samples from randomly selected hospital outpatients. Conversely, it is unusual not to observe a strong positive CPE in cultures from patients with otherwise unexplained neurological or behavioral disorders.

The stealth virus CPE is best characterized by the formation of foci of enlarged, rounded cells, often with cell fusion (syncytia). Proliferation foci of affected cells can occasionally be seen. The actual appearance of the CPE differs between cultures and is best followed by repeated examination of individual cultures by the same observer. The CPE can be transferred to fresh cultures. Positive cultures can be further examined by staining cell smears or sectioned cell pellets using the patient's and other sera. Electron microscopic studies can also be performed. Cell derived DNA and RNA can also be used for molecular characterization. A series of PCR primer sets based on previously characterized stealth viruses can be used to screen for virus-derived DNA and RNA sequences. The primers can also be used to test for DNA and RNA dependent polymerases. Finally, the viral cultures can be used to test the effects of various anti-viral therapies.

As noted above, stealth viral infections are not necessarily confined to the brain and indeed blood samples are routinely used for stealth viral cultures. Other serological signs of viral infections can include unusually high levels of anti-herpesvirus antibodies. This may reflect the presence of the stealth virus or the two-way cross stimulation that can be seen between stealth adapted viruses and conventional herpesviruses. Broadly reactive herpesvirus primers can also be used in low stringency PCR based assays on DNA and RNA directly isolated from the patient's blood [17]. Other primer sets have been shown to cross react with several stealth virus isolates in low stringency PCR assays. Cloning and sequencing of the PCR products can be used to design more specific primer sets. The possible role of stealth adapted herpesviruses in secondary activation of parvo- and papovaviruses can also be assessed using serological and molecular probe based assays for these agents.

\section{Stealth Adapted Viral Infection}

Stealth viruses have been isolated from blood and CSF of patients with a spectrum of illnesses with neurological and neuropsychiatric manifestations [17]-[22]. The clinical diagnoses have included autism and attention deficit learning disorders in children, CFS, fibromyalgia, Gulf war syndrome and depression in adults, and dementia/Alzheimer's disease in the elderly. Severe acute encephalopathy and major psychotic reactions have also been associated with positive stealth adapted viral infections. The clinical diversity seen in stealth viral infected patients may relate to the predominant areas of the brain that are infected as well as the timing and intensity of the infection. Stealth viruses from humans have induced acute neurobehavioral diseases in experimental animals [23] accompanied by similar histological and electron microscopic changes as seen in brain biopsies of infected humans.

\section{Histopathology}

The predominant histological characteristic in both humans and in the animal model is the presence of occasional cells with distinctly vacuolated, lipid-rich cytoplasm and distorted abnormal nuclei [18] [22] [23]. The affected cells may show varying granules positive with periodic acid Schiff (PAS) stain. Deposited material can also accumulate around small blood vessels, possibly impairing gas exchange and nutrient delivery. The marked vacuolization seen in some biopsies is certainly suggestive of Creutzfeld Jacob prion disease [22].

Animal studies confirmed that the cellular changes were not confined to the brain but that signs of infection could be found in various organs throughout the body [23]. Nonetheless, the predominant clinical manifestations in the animals were neurobehavioral, consistent with the unique susceptibility of the brain to limited damage. 


\section{Clinical Manifestations of Illnesses Seen in Certain Stealth Adapted Virus Infected Patients}

Numerous articles have described the protean clinical manifestations of major neuropsychiatric illnesses and conditions such as CFS and autism. This section is intended to highlight some of the clinical insights from extensive culturing of stealth adapted viruses from patients.

Stealth viral culture positive patients, whether presenting with a psychiatric or neurological illness, will not uncommonly report symptoms attributed to illnesses occurring elsewhere in the body. In some cases, such as in low back pain, the essence of the illness is a lowered pain threshold; more than the severity of the musculoskeletal changes. Similarly, pelvic pain can have a strong central nervous system component. Clinicians can err in over treating the localized area of pain without due regard to the underlying hypersensitivity of the patient's nervous system. The treated disease will likely recur or be replaced by an equally disabling painful condition occurring elsewhere in the body.

Various cardiovascular diseases, affecting the heart and/or peripheral circulation, can be ascribed to dysregulation by an impaired autonomic nervous system. Postural hypotension occurring in CFS patients is well described. An interesting illness encountered in some patients is erythromelalgia, in which inappropriate shunting of blood via opened arteriovenous connections compromises the capillary circulation with resulting pain [24].

The autonomic nervous system also controls aspects of gastrointestinal functioning and this can account for dysphagia and irritable bowel syndrome. It may also contribute to malabsorption, leaky gut syndrome and dysbiosis (abnormal gut flora). Bacteria sequences have been identified in some stealth adapted virus cultures [25]. This is consistent with some of these viruses being able to pass within bacteria. Indeed, atypical bacteria have been seen in fecal cultures of some CFS patients. Stealth adapted virus infection of the gut bacteria could, therefore, also contribute to dysbiosis.

Another diagnosis experienced by stealth adapted virus infected patients is delusional parasitosis [26]. Patients can produce pigmented particles with striking electrostatic properties, which the patients can easily mistake for living movements. When detected in the hair, the particles have also been misidentified as lice. The mother of a child reported severe mental deterioration requiring institutionalized care, after the anti-lice medication, Lindane, had been applied to her daughter's scalp and the house fogged with an insecticide.

A patient from whom strikingly positive cultures were repeatedly observed had numerous lipomas, which she said tended to come in episodes and slowly resolve. Her diagnosis was Dercum's disease, which is a condition largely unknown in conventional medicine [27]. This disease can be likened to periodic outbreaks of shingles. The difference is that instead of vesicular lesions developing in the skin, localized areas of excessive subcutaneous lipids are being produced; sometimes distributed within a single dermatome. Virus induced overproduction of intracellular lipids can explain liver steatosis seen in some stealth adapted virus infected patients. Using the same reasoning, intracellular lipids can inhibit glucose transport into cells, rendering cells somewhat insulin resistant. Obesity may result from the unsightly but possibly necessary disposal of lipids overproduced by virus infected parenchymal organs.

Virus infections can also provoke autoimmune reactions, especially to cellular DNA, as in systemic lupus erythematosus. Autoantibody production against mitochondrial phospholipids can lead to hypercoagulation, while antibodies to clotting factors can lead to excessive bleeding. Hashimoto's disease and Graves' disease can occur with auto-antibodies against thyroid antigens. Autoimmunity can also be directed to the nervous system, as can be seen with the extension of an apparent CFS to multiple sclerosis. Cases of limbic encephalopathy [28] can also be explained as an autoimmune response to neurotransmitters or to their receptors. Antibodies may also form against the virus, since many more virus antigens evoke humoral immunity than the relatively few antigens targeted by the cellular immune system. These antibodies can explain the vasculitis occurring in some stealth adapted virus culture positive patients [19]. The immune system itself can be directly damaged by virus infection leading to immune dysregulation.

An exception to the statement that limited localized damage is relatively less significant outside of the nervous system, is the occurrence of malignancy. Upon inquiry, breast cancer patients not uncommonly report on fatigue for years prior to the detection of their cancer and even after its removal. Multiple myeloma patients commonly have a prior history of neuropsychiatric illnesses. The consistent finding of positive cultures for stealth adapted viruses in multiple myeloma patients was confirmed in a double blind study [29]. Direct evidence for stealth adapted viruses was also obtained by using the PCR assay in patients with salivary tumors [30]. A patient 
with strikingly positive virus cultures had a glioblastoma, which is consistent with published evidence linking CMV to this tumor.

Virus infection of germ cells can potentially lead to genetic disorders in offspring. This has been noted in some patients with a genetic abnormality, not present as a somatic mutation in either of the parents. CMV is especially prone to infect the gonads and may induce genetic change in germ cell even prior to fertilization. This could account for the circumstances in which a de novo genetic disease occurs in children in whom one or more parent was stealth adapted virus infected.

\section{Transmission of Stealth Adapted Virus Infections}

The occurrence of family illnesses of presumptive infectious origin has been noted on several occasions. To cite one of these families: The mother openly declared she had CFS. She believed her husband also had the illness but was in denial since he was still required to work. The woman's mother was diagnosed with Parkinson's disease, while her son was diagnosed as schizophrenic. Within the family, they all recognized a basic similarity of their illnesses. Several other families with differing illnesses but all of presumptive infectious origin have been seen.

Community wide transmission of stealth adapted virus induced illnesses can easily explain many of the reported outbreaks of CFS-like illnesses. One such epidemic occurred in 1996 in the Mohave Valley area of Arizona and adjoining town of Needles, California [21]. The nation's blood and blood products supply is also an expected mode of transmission of stealth adapted viruses. Indeed, CDC was informed of positive stealth adapted virus cultures from $10 \%$ of blood donations collected in 2002 from the University of California Irvine.

Occupational exposure is also a potential risk factor, especially in individuals likely to come into close contact with others [31]. This group includes healthcare providers, schoolteachers, prison guards, etc. Avoiding becoming stealth adapted virus infected is of special concern to women anticipating pregnancy. A difficult issue, which also applies to conventional CMV infection, is the risk of daycare facilities and of pediatricians' medical offices in spreading infection to other children. Freshly infected children can expose their mother to the virus prior to or during her next pregnancy.

\section{Summary}

The specialization of medicine has focused attention on disorders that are essentially restricted to a single organ system. Multi-system diseases tend to fall outside the purview of most physicians and a balanced, comprehensive approach to their assessment is often lacking. The clinical evaluation of patients with a stealth viral induced encephalopathy should not be confined to disorders of brain function. Rather, the clinical evaluation should include seeking evidence for viral involvement of additional organs, as would be expected for a systemic virus infection. Efforts should also be undertaken to restrict the likely transmission of stealth adapted virus infections within families, certain occupations and whole communities.

\section{References}

[1] American Psychiatric Association (2013) Diagnostic and Statistical Manual of Mental Disorders. 5th Edition (DSM-5), Amer Psychiatric Pub Inc., Arlington.

[2] Noshpitz, J.D. and Coddington, R.D. (1990) Stressors and the Adjustment Disorders. John Wiley and Sons, New York.

[3] Tselis, A. and Booss, J. (2003) Behavioral Consequences of Infections of the Central Nervous System: With Emphasis on Viral Infections. Journal of the American Academy of Psychiatry and the Law, 31, 289-298.

[4] Ances, B.M. and Ellis R.J. (2007) Dementia and Neurocognitive Disorders Due to HIV-1 Infection. Seminars in Neurology, 27, 86-92. http://dx.doi.org/10.1055/s-2006-956759

[5] Torrey, E.F. (1988) Stalking the Schizovirus. Schizophrenia Bulletin, 14, 223-229. http://dx.doi.org/10.1093/schbul/14.2.223

[6] Tyler, K.L. (2004) Herpes Simplex Virus Infections of the Central Nervous System: Encephalitis and Meningitis, including Mollaret's. Herpes, 11, Suppl. 2, 57A-64A.

[7] Rhoades, R.E., Tabor-Godwin, J.M., Tsueng, G. and Feuer, R. (2011) Enterovirus Infections of the Central Nervous System. Virology, 411, 288-305. http://dx.doi.org/10.1016/j.virol.2010.12.014

[8] White, D.O. and Fenner, F.J. (1994) Medical Virology. 4th Edition, Academic Press, San Diego. 
[9] Carrasco, L. (1987) Mechanisms of Viral Toxicity in Animal Cells. CRC Press, Boca Raton.

[10] de la Torre, J.C., Borrow, P. and Oldstone, M.B. (1991) Viral Persistence and Disease: Cytopathology in the Absence of Cytolysis. British Medical Bulletin, 47, 838-851.

[11] Zinkernagel, R.M. (1997) Immunology and Immunity Studied with Viruses. Ciba Foundation Symposium, 204, 105125.

[12] Martin, W.J. (1994) Stealth Viruses as Neuropathogens. College of American Pathologist’s Publication. CAP Today, 8, 67-70.

[13] Martin, W.J. (1999) Stealth Adaptation of an African Green Monkey Simian Cytomegalovirus. Experimental and Molecular Pathology, 66, 3-7. http://dx.doi.org/10.1006/exmp.1999.2248

[14] Burnet, F.M. (1959) The Clonal Selection Theory of Acquired Immunity. Vanderbilt University Press, Nashville. http://dx.doi.org/10.5962/bhl.title.8281

[15] Wills, M.R., Carmichael, A.J., Mynard, K., Jin, X., Weekes, M.P., et al. (1996) The Human Cytotoxic T-Lymphocyte (CTL) Response to Cytomegalovirus Is Dominated by Structural Protein pp65: Frequency, Specificity, and T-Cell Receptor Usage of pp65-Specific CTL. Journal of Virology, 70, 7569-7579.

[16] Martin, W.J. (1996) Genetic Instability and Fragmentation of a Stealth Viral Genome. Pathobiology, 64, 9-17. http://dx.doi.org/10.1159/000164000

[17] Martin, W.J., Zeng, L.C., Ahmed, K. and Roy, M. (1994) Cytomegalovirus-Related Sequences in an Atypical Cytopathic Virus Repeatedly Isolated from a Patient with the Chronic Fatigue Syndrome. American Journal of Pathology, 145, 441-452.

[18] Martin, W.J. (1996) Severe Stealth Virus Encephalopathy Following Chronic Fatigue Syndrome-Like Illness: Clinical and Histopathological Features. Pathobiology, 64, 1-8. http://dx.doi.org/10.1159/000163999

[19] Martin, W.J. (1996) Stealth Viral Encephalopathy: Report of a Fatal Case Complicated by Cerebral Vasculitis. Pathobiology, 64, 59-63. http://dx.doi.org/10.1159/000164009

[20] Martin, W.J. (1996) Simian Cytomegalovirus-Related Stealth Virus Isolated from the Cerebrospinal Fluid of a Patient with Bipolar Psychosis and Acute Encephalopathy. Pathobiology, 64, 64-66. http://dx.doi.org/10.1159/000164010

[21] Martin, W.J. and Anderson, D. (1997) Stealth Virus Epidemic in the Mohave Valley. Initial Report of Viral Isolation. Pathobiology, 65, 51-56. http://dx.doi.org/10.1159/000164103

[22] Martin, W.J. and Anderson, D. (1999) Stealth Virus Epidemic in the Mohave Valley: Severe Vacuolating Encephalopathy in a Child Presenting with a Behavioral Disorder. Experimental and Molecular Pathology, 66, 19-30. http://dx.doi.org/10.1006/exmp.1999.2237

[23] Martin, W.J. and Glass, R.T. (1995) Acute Encephalopathy Induced in Cats with a Stealth Virus Isolated from a Patient with Chronic Fatigue Syndrome. Pathobiology, 63, 115-118. http://dx.doi.org/10.1159/000163942

[24] Ljubojević, S., Lipozencić, J. and Pustisek, N. (2004) Erythromelalgia. Acta Dermatovenerologica Croatica, 12, 99105.

[25] Martin, W.J. (1999) Bacteria Related Sequences in a Simian Cytomegalovirus-Derived Stealth Virus Culture. Experimental and Molecular Pathology, 66, 8-14. http://dx.doi.org/10.1006/exmp.1999.2239

[26] Martin, W.J. (2005) Alternative Cellular Energy Pigments Mistaken for Parasitic Skin Infestations. Experimental and Molecular Pathology, 78, 212-214. http://dx.doi.org/10.1016/j.yexmp.2005.01.007

[27] Hansson, E., Svensson, H. and Brorson, H. (2012) Review of Dercum's Disease and Proposal of Diagnostic Criteria, Diagnostic Methods, Classification and Management. Orphanet Journal of Rare Diseases, 7, 23. http://dx.doi.org/10.1186/1750-1172-7-23

[28] Ramanathan, S., Mohammad, S.S., Brilot, F. and Dale, R.C. (2014) Autoimmune Encephalitis: Recent Updates and Emerging Challenges. Journal of Clinical Neuroscience, 21, 722-730.

[29] Durie, B.G., Collins, R.A. and Martin, W.J. (2000) Positive Stealth Virus Cultures in Myeloma Patients: A Possible Explanation for Neuropsychiatric Co-Morbidity. Blood, 96, 29.

[30] Gollard, R.P., Mayr, A., Rice, D.A. and Martin, W.J. (1996) Herpesvirus-Related Sequences in Salivary Gland Tumors. Journal of Experimental \& Clinical Cancer Research, 15, 1-4.

[31] Joseph, S.A., Béliveau, C. and Gyorkos, T.W. (2006) Cytomegalovirus as an Occupational Risk in Daycare Educators. Paediatrics \& Child Health, 11, 401-407. 


\section{Abbreviation}

SCMV-African green monkey simian cytomegalovirus,

hCMV-human cytomegalovirus,

CPE—cytopathic effect,

PCR_-polymerase chain reaction,

CSF_cerebrospinal fluid. 